\title{
Gabor Transform Based Glioma Brain Tumor Detection Using Neural Networks
}

\author{
S. Deivasigamani ${ }^{\# 1}$, Vissakam Ganesan ${ }^{\# 2}$, Dhinakar Pazhani ${ }^{\# 3}$, \\ Manickam Ramasamy ${ }^{* 4}$, Umayal $^{\# 5}$ \\ \# Faculty of Engineering and Computer Technology, AIMST University \\ 08100 jalan Semeling-Bedong, Kedah Darul Aman, Malaysia \\ ${ }^{1}$ deivasigamani@aimst.edu.my \\ ${ }^{*}$ Faculty of Engineering, Technology \& Built Environment, UCSI University \\ 5600 Cheras, Kuala Lumpur, Malaysia
}

\begin{abstract}
Detection and segmentation of abnormal regions in brain MRI images is a complex task due to its similarity with its surrounding pixels. In this paper, brain tumors are detected and segmented using neural network classifier in brain MRI images which are available from open access dataset. The proposed methods have the following stages as preprocessing, Gabor transform, feature extraction, NN classification and morphological based segmentation. The spatial domain image is converted into multi resolution image using Gabor transform and further features are extracted from this Gabor transformed image. These features are trained and classified using feed forward back propagation neural network classification approach. Then, morphological segmentation process is applied over the classified abnormal image in order to segment the tumor regions in brain MRI image. The performance of the tumor segmentation method is analyzed in terms of sensitivity, specificity, positive predictive value, negative predictive value and accuracy. The proposed method stated in this work obtains $97.62 \%$ of sensitivity, $99.98 \%$ of specificity, $\mathbf{9 9 . 0 6} \%$ of positive predictive value, $99.41 \%$ of negative predictive value and $99.40 \%$ of accuracy with respect to ground truth images.
\end{abstract}

Keyword - Brain tumours, Gabor transform, Glioma, MRI, Neural network.

\section{INTRODUCTION}

Brain acts as the focal point of focal sensory system in human beings. The human brain contains 50-100 billion neurons in its system forming the largest colossal system. Tumor is formed in brain by a group of abnormal growth in cells within the brain or around it. Tumors of the brain may be benign or malignant. Malignant may be cancerous and can be treated if detected at an earlier stage. It is classified into primary and secondary stages of tumor. Benign tumors are not dangerous as it does not spread to other parts, rather malignant tumors are very dangerous as it spreads to other brain tissues very faster and affects the normal operation of the central nervous system. The location of tumors within brain structure is a tedious task as the brain is composition of complex cells and tissues. The exact location of tumor is essential for giving proper treatment to remove the damaged cells. Magnetic Resonance Imaging (MRI) is a technique to analyze the tumor location within the brain and gives an exact image of the tumor. The cells in brain MRI image are categorized into the following as Gray Matter (GM), White Matter (WM), and Cerebro Spinal Fluid (CSF). These regions are important in tumor classification, as to locate the tumor presence in which region. According to surveys, tumors form the second main reason for cancer-related mortals in male who are younger than 20 years. Similarly, it is the fifth reason for cancer-related deaths in females who are aged between 20 and 39. The most widely recognized primary brain tumors are,

- Gliomas

- Meningiomas

- Pituitary adenomas

- Nerve sheath tumors

Every tumor has different qualities [1-3] that has to be analyzed for its hazardous feature, its developmental way and to help the medicinal group to formulate the cure for such tumors. Some of them are as follows: Anaplasia or dedifferentiation; loss of separation of cells and veins, a normal for anaplastic tumor tissue. Anaplastic cells are cells without proper control and very last stage of damaged cell structure. These cells are present with high atomic to-cytoplasmic proportions and are having multiple nuclei. The anaplastic cells have a multi-core structure, i.e. more number of cores than usual. The cells follow a dedifferentiation process to end up with anaplasia and the cells lose their ordinary structure after differentiation. This is called neoplasia. The neoplastic cell duplicates itself due to cancer causing organisms and follows a rapid separation. 
The next one is atypia, in which the cells divide uncontrollably which forms a threat to life. Since the division of cells is not a danger, even though it divides rapidly, a pressure is developed in the cranial space due to neoplasm and might cause damage to brain tissues and increases the cranial weight. Increase in intracranial mass directly affects the impact on tumor cells, increase in fluid volume and other timely side effects.

Putrefaction is the process of damaging of brain cells, caused by external factors, such as, diseases, poison or any type of injury. Immediate after the dead cells are present, the necrotic cells have to clear the dead cells, but in this case, they receive wrong signs which keep phagocytes from discarding these dead cells, thereby giving command to develop dead tissues. There creates a less supply of oxygen to the brain tissues called venous hypoxia, or the unavailability of sufficient oxygen to specific regions of the brain. It happens when a tumor cell utilizes the adjacent veins for its blood supply and the neoplasm becomes unavailable for providing supplements to the brain tissue. After occurrence of neoplasm, there might be less volume of intracranial cavity, causing harm to the brain.

\section{LITERATURE SURVEY}

Bhagwat et al [4] exhibited a work that combines K-means, Fuzzy C-means and Hierarchical grouping to determine the brain tumor. The author proposed the combination of these algorithms over MRI brain image in various formats. The DICOM format provides more data in the implementation process. Their method included various stages- pre-processing, highlight extraction, segmentation and classification. The K-means algorithm performed better than other clustering techniques. Back Propagation Neural Network (BPNN) was utilized for the classification stage which accurately detected the tumor into benign or malignant.

Magdi et al [6] utilized a shrewd Model for brain tumor analysis from MRI pictures which comprise of three unique stages, for example, pre-processing, Feature extraction and segmentation. In the Pre-processing step, filters are used to enhance the MRI image using edge detection, then features are extracted and finally, Principal Component Analysis (PCA) is used to detect the tumor region. The Back Propagation Neural Network (BPNN) is applied to accurately classify the tumor region and its malignancy from the brain image. N.Senthil kumaran et al [7] exhibited a half and half technique for white issue partition from MRI brain picture that comprise of three stage. To start with stage is to preprocess a picture for division, second stage is to fragment a picture utilizing granular harsh set and third stage is to isolate white issue from sectioned picture utilizing fluffy sets This technique was contrasted and mean move calculation and it was discovered that half and half division performs better outcome.

Sivaramkrishnan et al. [8] displayed a Fuzzy C-mean (FCM) based brain tumor detection methodology. In their method, the algorithm was implemented to find the centroids of a cluster and repeated for each cluster. This method is easy in finding the centroid and provides faster segmentation. The histogram technique was applied to enhance the contrast and PCA to detect the tumor region.

Atiq Islam et al. [9] used a multi - determination fractal demonstrate called the multifractional Brownian movement $(\mathrm{mBm})$ for the distinguishing proof of tumor district, which are of various sizes and surfaces. The Jaccard scores created by this technique require change.

A picture can be portioned by numerically illuminating an Partial Differential Equation (PDE)- based strategy [10]. Bend proliferation is a famous system in PDE based technique, by which various applications identified with question extraction, protest following, stereo recreation, and so forth are performed. The prime thought of utilizing this strategy is to discover an underlying bend towards the most minimal capability of a cost work, where its portrayal relates the assignment to be handled. For the greater part of the opposite issues, minimization of the cost work is of much significance and it proposes certain smoothness imperatives on the arrangement, which can be spoken to as geometrical limitations on the advancing bend.

Lagrangian procedures rely upon parameterization of the form as indicated by certain inspecting systems and after that deliver component esteems as per pictures and inside terms [11]. Such methods are sufficiently quick and proficient in creating division comes about; in any case, the first "absolutely parametric" detailing is for the most part scrutinized for its constraints in picking the examining procedure, topology changes, tending to issues in higher measurements, the inner geometric properties of the bend, and so forth. As of late, proficient "discretized" definitions have been developed to address the impediments brought about amid high effectiveness support. In the above said cases, vitality minimization is for the most part sorted out utilizing steepest- gradient descent methods.

Picture mosaicking for assessment of MR brain tissue variations from the norm division consider [12], fragments the tumor district just in T1 weighted pictures. The investigation of assessing the effect of various factors on voxel-based grouping techniques for ADNI auxiliary MR brain pictures performs division upon T1 weighted pictures. Dormancy acts to be a noteworthy difficulty in proposals voxel based order strategies. Division of different sclerosis sores in brain MR pictures utilizing Spatially Constrained Possibilistic Fuzzy Cmeans Classification approves division of T1 and T2 weighted pictures utilizing FCM grouping strategy. 
Possibilistic FCM creates better segmentation. Probabilistic FCM creates better segmentation accuracy, which is confined to small and tiny lesions present in the input MR brain images.

Automatic brain extraction methods for T1 magnetic resonance images using region labeling and morphological operations [13] perform segmentation only upon T1 weighted images using BEM (Brain Extraction Methods). Tissues of brain are segregated from cerebrospinal fluid and skull regions, automatically.

\section{PRoposed System}

In this paper, brain tumors are detected and segmented using neural network classifier in brain MRI images which are available from open access dataset. The proposed methods have the following stages as preprocessing, Gabor transform, feature extraction, NN classification and morphological based segmentation. The proposed brain tumor detection and analysis methodology is shown in Fig 1.

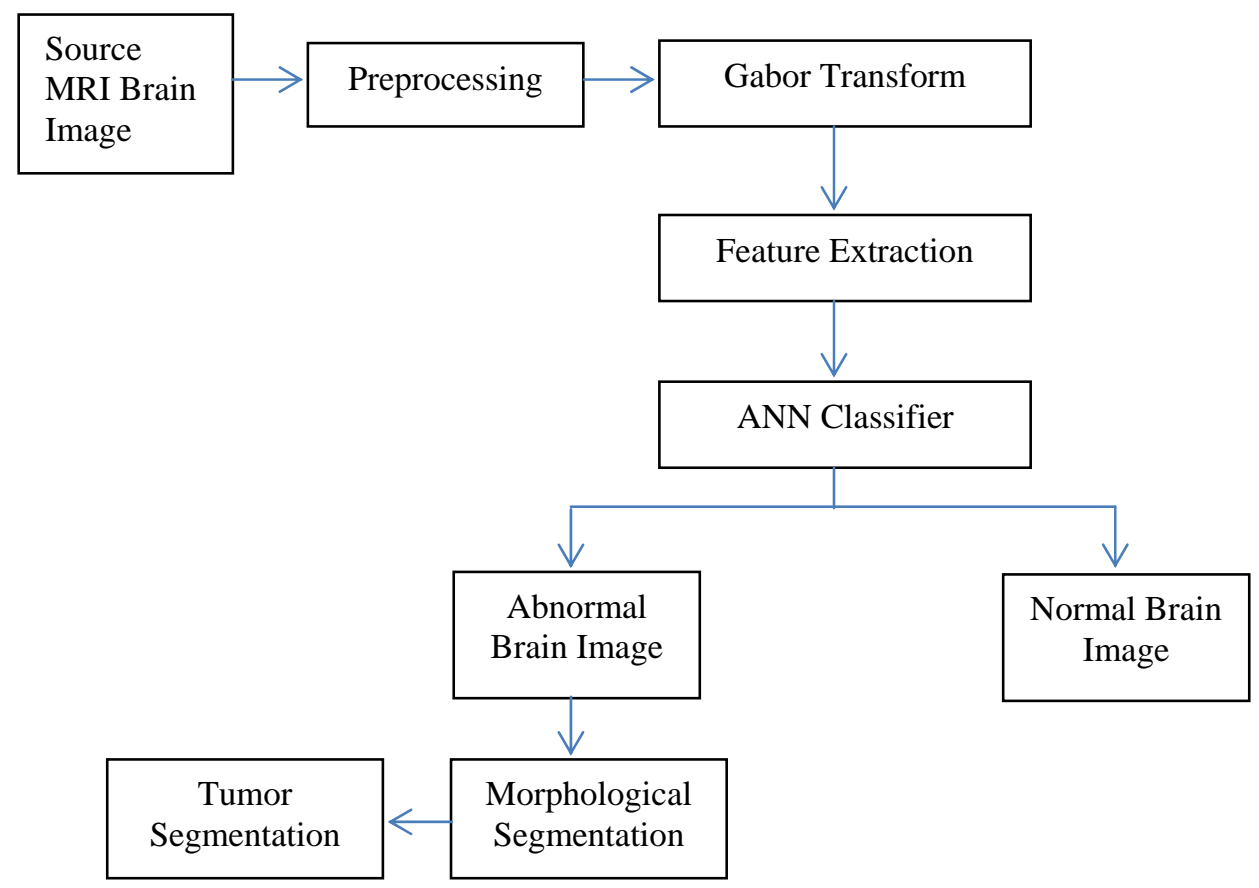

Fig. 1. Proposed brain tumor detection and analysis methodology

\section{A. Gabor Transform}

The pre-processed brain MRI image is one kind of transformation which is in spatial resolution. The pixels in spatial resolution format cannot be modified and processed further feature extraction steps. In this conjunction, the spatial resolution pixels in pre-processed image are transformed into multi class resolution pixels' format. The pixels in this multi class resolution transformed image can be modified and processed under any circumstances. Hence, Gabor transform is used on the pre-processed brain MRI image in order to obtain the multi class resolution pixel format. In this paper, 2D- Gabor transform with its Gaussian Gabor kernels are used for convolving the spatial resolution brain MRI image with Gabor kernels.

In this paper, the following Gaussian Gabor kernel is used for convolution process between spatial resolution image and kernel and it is defined as,

$$
\varphi_{p, q}=\frac{\mathrm{P} 2}{\sigma^{2}} \exp \left(-\frac{\mathrm{P} 2}{2 \sigma^{2}}\right) \times\left[\exp (i * \mathrm{p} * z)-\exp \left(-\frac{s^{2}}{2}\right)\right]
$$

The scale of the Gabor kernel in this equation is represented by ' $\mathrm{s}$ ' and the pixel rate is varied based on this scale factor. It is ranges between 0 and 10. For low rate pixel conversion, the lower value of scale factor is selected. For high rate pixel conversion, the higher value of scale factor is selected. The orientation of the Gabor kernel in this equation is represented by ' $\sigma$ ' and it is based on pixel angle with respect to its location in the preprocessed brain MRI image. The orientation of each pixel in the image is varied between 0 and 360 degrees. ' $\mathrm{P}$ ' in this equation is noted as pointing wave factor with respect to its position of pixels (z) in the brain MRI image. This Gabor kernel is convolved with pre-processed brain MRI image for all orientation of pixels.

\section{B. Feature Extraction}

The process next to Gabor transformation is feature extraction. The changes or variations in multi class pixel transformed Gabor image are detected using feature extraction process. In this paper, energy features such as GLCM features and LBP features are derived from the Gabor transformed brain MRI image. 
1) GLCM Features: The energy distribution is equal throughout the brain regions in normal brain MRI image. It is not equally distributed in brain regions in abnormal brain MRI image. This property is used for differentiating the normal brain MRI image from abnormal brain MRI image using the feature called as GLCM feature. The GLCM matrix is formed for the entire image with respect to 45-degree orientation of pixels. The normalized symmetrical GLCM features extracted are,

$$
\begin{aligned}
& \text { Contrast }=\sum\left(|x-y|^{2} \times p(x, y)\right) \\
& \text { Energy }=\sum p(x, y)^{2} \\
& \text { Homogeneity }=\frac{\sum p(x, y)}{1+|x-y|} \\
& \text { Correlation }=\sum(x-\mu x)(j-\mu j) \frac{p(x, y)}{[\sigma i . \sigma j]}
\end{aligned}
$$

In these GLCM feature extraction equations, the index of GLCM matrix is represented as ' $x$ ' and ' $y$ '.

2) Local Binary Pattern: The LBP operator was introduced for texture classification. Given a center pixel in the image, the LBP value is computed by comparing its gray value with its neighbours, as shown in the equation below

$$
L B P_{P, R}=\sum_{p=1}^{P} 2^{(p-1)} X f_{1}\left(g_{p}-g_{c}\right) \text {, where } f_{1}(x)=\left\{\begin{array}{c}
1, x \geq 0 \\
0, \text { else }
\end{array}\right.
$$

3) Classification: The SVM classification approach was used for the past decades for detecting and classifying the brain MRI image into its various cases. The feedback path was not available in SVM classification approach which degraded the classification process. This drawback is overcome by using NN classification approach on brain MRI image. The NN classifier is used to differentiate the normal brain MRI image from abnormal one and further Adaptive Neuro Fuzzy Inference System (ANFIS) classifier is used to classify the abnormal brain MRI image into benign and malignant. In this paper, feed forward back propagation NN classification algorithm is used with five hidden layers and unique input and output layers.

\subsection{Feed forward network}

The logic behind this Feed forward is simple and easy to understand. The neural network starts from the input layer and it computes the output by calculating and updating the weights of the each edge connecting the layers. This output will be compared with the desired output. If there is a huge alteration between the wanted yield and the calculated yield, then the error is calculated and the process is repeated again and again until the error is negligibly small. The neural network has to be run and trained with the input set of samples. The weight setting and updating are also essential part of neural network, because when it is updated each time, the results are improved and the error is minimized. Activation function shows a significant part in creating non linearity in the hidden layers. Feed forward algorithm is one of the major neural network algorithms, which to describe as the following four steps; they are

- $\quad$ Feed forwarding

- BP to output layer

- BP to hidden layer

- Weight updating

\section{Morphological Operation Based Tumor Segmentation}

The malignant brain MRI image is differentiated from benign brain image for further detection and segmentation of tumor regions in brain MRI image. In this paper, morphological functions such as opening and closing with respect to erosion and dilation are used. The mathematical presentation of morphological functions is explained in the following equations with respect to its structuring element $(\mathrm{H})$. The pixels in classified image are enhanced with respect to its outlier location using the process called as morphological dilation process.

Dilation:

$$
(\mathrm{I} \oplus \mathrm{H})(\mathrm{x}, \mathrm{y})
$$

The pixels in classified image are decreased with respect to its inner location using the process called as morphological erosion process.

Erosion:

$$
(\mathrm{I} \ominus \mathrm{H})(\mathrm{x}, \mathrm{y})
$$

Fig. 2(a) shows the source brain images, Fig. 2(b) shows the ground truth images and Fig. 2(c) shows the tumor region segmentation by proposed method and Fig. 2(d) shows the tumor region segmentation by conventional method (Bahadure et al. [14]). 

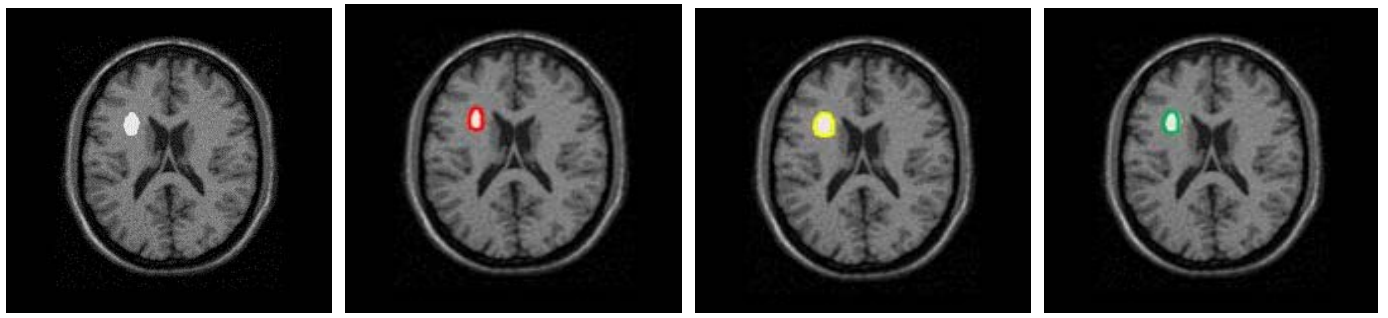

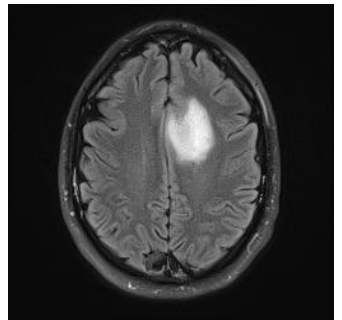

(a)

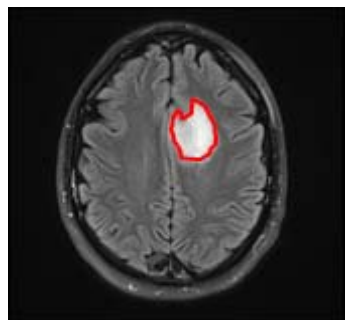

(b)

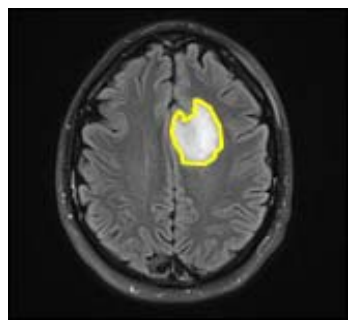

(c)

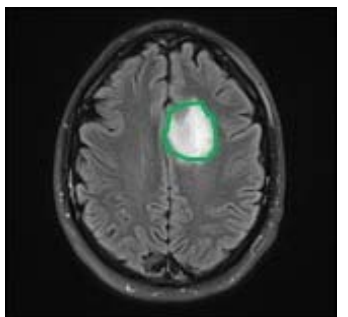

(d)

Fig. 2. (a) Source brain images; (b) Ground truth images; (c) Tumor region segmentation by proposed method; (d) Tumor region segmentation by conventional method (Bahadure et al. [14])

\section{RESULTS AND DISCUSSIONS}

In this paper, Multimodal Brain Tumor Image Segmentation (BRATS) dataset (Brainweb Database, $<$ http://www.bic.mni.mcgill.ca/brainweb>) [15] is used for the detection and segmentation of tumor region in brain images. The modality of the brain images in this dataset is Magnetic Resonance Imaging (MRI). This dataset also contains ground truth images which are the images obtained from expert radiologist. In this paper, the images from this open access dataset are grouped into training and testing set. Numerous simulations were performed and the results are given as follows. The original image used for our experimentation is shown in Figure 6(a). The proposed method segments the cancer regions from the feature sets and the abnormal regions are marked in the cervical image in blue color.

The features such as GLCM, Gray level features are extracted from the pre-processed cervical image and then processed with our proposed cervical cancer detection algorithm. The features or patterns extracted for GLCM features are clearly tabulated in Table 2. Finally, the ANFIS classifier classifies the tumor region and the resultant tumor segmented image is shown in Figure 6(c).

The performance of brain cancer region segmentation is analyzed with the following parameters:

$$
\begin{aligned}
& \text { Sensitivity }[\mathrm{Se}=\mathrm{TP} /(\mathrm{TP}+\mathrm{FN})] \\
& \text { Specificity }[\mathrm{Sp}=\mathrm{TN} /(\mathrm{TN}+\mathrm{FP})] \\
& \text { Accuracy }[\mathrm{Acc}=(\mathrm{TP}+\mathrm{TN}) /(\mathrm{TP}+\mathrm{FN}+\mathrm{TN}+\mathrm{FP})]
\end{aligned}
$$

These parameters are evaluated for a set of images and listed in Table 1, where, TP and TN is correctly detected normal pixels in classified and segmented brain MRI image, FP and FN is mistakenly detected abnormal tumor pixels in classified and segmented brain MRI image.The parameters, Se and Sp define the ratio of wellclassified brain cancer region and non-cancerous region, respectively. Acc is the ratio of total well-detected and classified brain cancer region. Table I evaluates the result of performance parameters for the segmentation of cancer regions of brain and its performance comparisons with state of arts are depicted in Table III. The features or patterns extracted for GLCM features are clearly tabulated in Table II.

Table I. Performance Analysis of Proposed Tumor Segmentation Method

\begin{tabular}{|c|c|}
\hline Performance Evaluation Parameters & Tumor segmentation results (\%) \\
\hline Sensitivity & 97.62 \\
\hline Specificity & 99.98 \\
\hline Positive Predictive Value & 99.06 \\
\hline Negative Predictive Value & 99.41 \\
\hline Accuracy & 99.40 \\
\hline
\end{tabular}


Table II. GLCM Features

\begin{tabular}{|c|c|c|}
\hline GLCM Features & Normal Region & Abnormal Region \\
\hline Energy & $1.55^{*} 10^{-5}$ & $1.625 * 10^{-5}$ \\
\hline Entropy & 1.1079 & 1.105 \\
\hline Autocorrelation & $1.618 * 10^{4}$ & $1.594 * 10^{4}$ \\
\hline Contrast & $1.12 * 10^{4}$ & $1.303 * 10^{4}$ \\
\hline
\end{tabular}

Table III. Performance Comparisons of Proposed Tumor Segmentation Method with Other State-of-the-Arts Methods

\begin{tabular}{|l|c|c|c|c|}
\hline \multirow{2}{*}{$\begin{array}{l}\text { Performance Evaluation } \\
\text { Parameters }\end{array}$} & \multicolumn{4}{|c|}{ Tumor Segmentation Results } \\
\cline { 2 - 5 } & $\begin{array}{c}\text { Proposed } \\
\text { Method }\end{array}$ & $\begin{array}{c}\text { Sreedhanya and } \\
\text { Pawar (2017) }\end{array}$ & $\begin{array}{c}\text { Bahadure et al. } \\
\text { (2017) }\end{array}$ & $\begin{array}{c}\text { Alfonse and } \\
\text { Salem (2016) }\end{array}$ \\
\hline Sensitivity & 97.62 & 93.1 & 97.1 & 96.37 \\
\hline Specificity & 99.98 & 94.6 & 94.2 & 95.28 \\
\hline Positive Predictive Value & 99.06 & - & - & - \\
\hline Negative Predictive Value & 99.41 & - & - & - \\
\hline Accuracy & 99.40 & 94.1 & 96.5 & 96.38 \\
\hline
\end{tabular}

\section{CONCLUSiON}

This paper proposes an efficient tumor detection and segmentation method using neural network classification method on brain MRI images. The spatial domain image is converted into multi resolution image using Gabor transform and further features are extracted from this Gabor transformed image. These features are trained and classified using feed forward back propagation neural network classification approach. Then, morphological segmentation process is applied over the classified abnormal image in order to segment the tumor regions in brain MRI image. The proposed method stated in this work obtains $97.62 \%$ of sensitivity, $99.98 \%$ of specificity, $99.06 \%$ of positive predictive value, $99.41 \%$ of negative predictive value and $99.40 \%$ of accuracy with respect to ground truth images.

\section{REFERENCES}

[1] O.P. Verma, M. Hammandlu, S. Susan, M. Kulkarni and P.K. Jain, “A simple single seeded region growing algorithm for color image segmentation using adaptive thresholding,” International Conference on Communication Systems and Network Technologies, (2011), pp. 500-503.

[2] A.Chinnu, “MRI Brain Tumor Classification Using SVM and Histogram Based Image Segmentation,” International Journal of Computer Science and Information Technologies, Vol. 6, (2015), pp.1505-1508.

[3] B. Gupta and S. Tiwari, “Brain Tumor Detection using Curvelet Transform and Support Vector Machine,” International Journal of Computer Science and Mobile Computing, Vol. 3, No. 4, (2014), pp. 1259-1264.

[4] K. Bhagwat, Dhanshri More, Sayali Shinde, Akshay Daga, Assistant Prof. Rupali Tornekar "Comparative Study of Brain Tumor Detection Using K-means, Fuzzy C means and Hierarchical Clustering Algorithms,” International Journal of Scientific \& Engineering Research, Vol. 2, No. 6, (2013), pp. 626-632.

[5] Monica subashini .M, Sarat Kumarn Sahoo, "Brain MR image Segmentation for Tumor Detection Using Artificial Neural Networks", International Journal of Engineering and Technology (IJET), ISSN: 0975-1024, Vol. 5, No. 2, (2013), pp. 925-930

[6] Magdi B.M Amien, Ahmed Abd-elrehman and Walla Ibrahim, “An Intelligant Model for Automatic Brain Tumor Diagnosis Based on MRI Images,” International Journal of Computer Applications (0975-8887) Vol. 72, No. 23, (2013), pp. 21-24.

[7] N.Senthil kumaran, R.Rajesh, C.Thilagavathy, "Hybrid Method for White Matter Separation In Brain Images Using Granular Rough Sets and Fuzzy Thresholding,” Proceedings of IEEE 17 International Conference on Image Processing, 2010, Hong Kong, 978-142447993, (2010), IEEE, pp. 3037-3040.

[8] A.Sivaramakrishnan, M.Karnan, “A Novel based Approach for Extraction of Brain Tumor in MRI Images Using Soft Computing Techniques”, International Journal of Advanced Research in Computer and Communication Engineering, Vol. 2, No. 4, (2013), pp. $1845-1848$

[9] Atiq Islam, Syed M. S. Reza and Khan M. Iftekharuddin, "Multifractal Texture Estimation for Detection and Segmentation of Brain Tumors”, IEEE Transactions on Biomedical Engineering, Vol. 60, No. 11, (2013), pp. 3204 - 3215.

[10] Caselles. V, Kimmel. R and Sapiro. G, Geodesic active contours, International Journal of Computer Vision, Vol. 22, No. 1, (1997), pp. 61-79.

[11] Kass. M, Witkin. A and Terzopoulos. D, Snakes, “Active contour models”, International Journal of Computer Vision, Vol. 1, No. 4, (1988), pp. 321-331.

[12] Shafaf Ibrahim, Noor Elaiza, Abdul Khalid and Mazani Manaf, Image Mosaicing for Evaluation of MRI Brain Tissue Abnormalities Segmentation Study, International Journal of Biology and Biomedical Engineering, Vol. 5, No. 4, (2011).

[13] Somasundaram. K and Kalaiselvi. T, “Automatic brain extraction methods for T1 magnetic resonance images using region labeling and morphological operations”, Computers in Biology and Medicine, Vol. 41, (2011), pp. 716-725.

[14] Bahadure NB, Ray AK, Thethi HP, "Image analysis for MRI based brain tumor detection and feature extraction using biologically inspired BWT and SVM,” Int J Biomed Imag. Vol. 2017, (2017), pp. 1-12.

[15] BRATS database, Available at: < http://www.bic.mni.mcgill.ca/ brainweb >

[16] Sreedhanya S, Pawar CS. An automatic brain tumor detection and segmentation using hybrid method. Int J Appl Inform Syst. 2017; 11:6-11.

[17] Alfonse M, Salem ABM. An automatic classification of brain tumors through MRI using support vector machine. Egypt Comp Sci J. 2016; 40:11-21. 


\section{AUTHOR PROFILE}

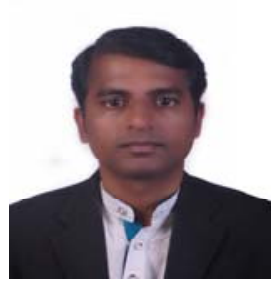

DEIVASIGAMANI. S received the B.Engg., M.Engg degrees in Electrical and Electronics Engineering, Applied Electronics from Thiagarajar College of Engineering, Thanthai Periyar Government Institute of Technology, Anna University, India in 2006 \& 2009 respectively. He is presently working as a Senior Lecturer cum Deputy Dean in the Faculty of Engineering and Computer Technology, AIMST University, Malaysia and doing $\mathrm{PhD}$ in the area of medical signal processing in Multimedia University, Malaysia. His research interests include signal and image processing in bio medical engineering and applications. His work has been documented in 10 papers in international journals and conferences and has presented invited and contributed talks at colleges and electronic industry. He is an IEEE member and registered Chartered Engineer with Engineering Council, United Kingdom.

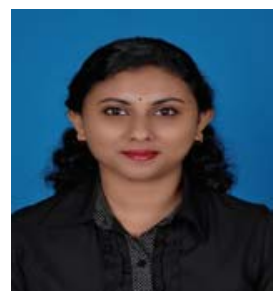

VISSAKAM GANESAN received Bachelor of Engineering (Hons.) in Electrical and Electronic Engineering from AIMST University, Kedah, Malaysia in 2018. She is an IEM student member.

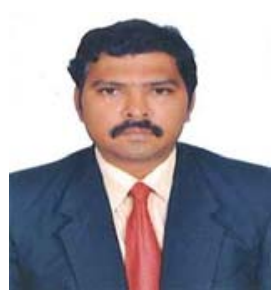

Dr. Dhinakar got his Undergraduation from Annamalai university in Electrical and Electronics Engineering in the year 1998. He went on to finish his masters in VLSI Design and there after $\mathrm{PhD}$ in Information and Communication Engineering from Anna university, Chennai. He is richly experienced in academic field and his areas of interest in research Covers semiconductor technology, soft error detection and smart grid. Currently, he is serving as a Lecturer in Aimst University, Malaysia.

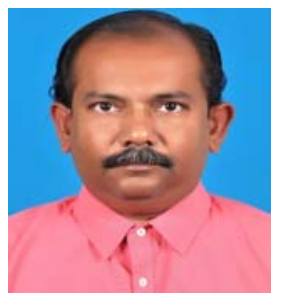

Manickam Ramasamy was born in India in 1968. He received the B.E. degree in Electronics and Communication from University of Madras, India in 1989, and M.Eng,Sc from Multimedia University, Malaysia in 2007. He is presently working as a Lecturer in Electrical and Electronics Department, UCSI University in Malaysia. His research interests include digital systems and image processing applications. He is an IET member and registered Chartered Engineer with Engineering Council, United Kingdom.

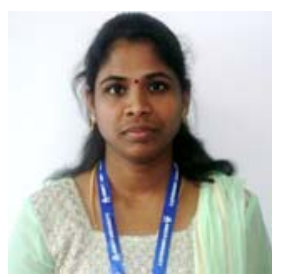

Umayal.V received B.E in Computer Science Engineering from Anna University Chennai, India in 2005. He received M.E in Embedded System Technologies from Anna University, Chennai, India in 2008. Her research interests are Embedded System Design, Cloud computing and Cryptography. 